aspects of the subject will be discussed at greater length elsewhere.

Research Laboratory,

C. H. BAMFORD

W. E. HANBY

Courtaulds, Ltd.

Maidenhead, Berks.

X-Ray Laboratory,

Courtaulds, Ltd.,

Coventry.

Sept. 14.

'Bamford, C. H., Hanby, W. E., and Happey, F., Nature, 164, 138 (1949).

'Huggins, M. L., Ann. Rev. Biochem., 11, 27 (1942).

Ambrose, E. J., and Hanby, W. E., Nature, 163, 483 (1949).

Astbury, W. T., Chem. and Indust., 60, 491 (1941).

'Astbury, W. T., Nature, 164, 439 (1949).

\section{High-Speed Recording by a Rotating-Mirror Method}

IN the course of a series of experiments on the propagation of transient stress waves in solids, we have developed an optical method of high-speed recording which does not appear to be described in the literature. The duration of the stress pulses is often of the order of 50 microsec., and the only feasible method of recording the phenomena optically is to use a stationary-film camera in conjunction with a mirror rotating at high speed, the recording film being fixed on a circular arc the centre of which lies on the axis of the mirror. If the radius of this are is $27 \mathrm{~cm}$., a writing speed of $3 \mathrm{~mm}$. per microsec. (measured on the film) will be obtained when the mirror rotates at 1,000 rev. per sec. Rotational speeds of this order of magnitude can be obtained most easily by means of the high-speed air turbines developed for ultracentrifuge work $^{1}$, and, in our camera, the rotating mirror consists of an octagonal, stainless steel mirror mounted on the rotor of a small turbine of this type.

The camera can be put to many uses, and a typical example of its application is the investigation of the propagation of torsional waves in steel cylinders; for this purpose, a minute optical flat was polished on the surface of the bar and the variation of the angular deflexion, $\theta$, of this flat with time, $t$, was recorded by reflecting a narrow pencil of light from it to the rotating mirror and thence to the recording film. A stress pulse consisting of torsional waves was initiated by firing a rifle bullet at a suitable point on a flat surface machined on one end of the bar, and a typical record obtained under these conditions is shown in the accompanying figure.

The lower trace is a datum line obtained when the bar was at rest. The upper trace shows the stress pulse reaching the recording mirror at $t=0$ and end. ing at $t=35$ microsec.; $\theta$ then remains constant until $t=60$ microsec., when the pulse returns to the recording mirror after reflexion at the free end of the bar. The reflected pulse ends at $t=95$ microsec.

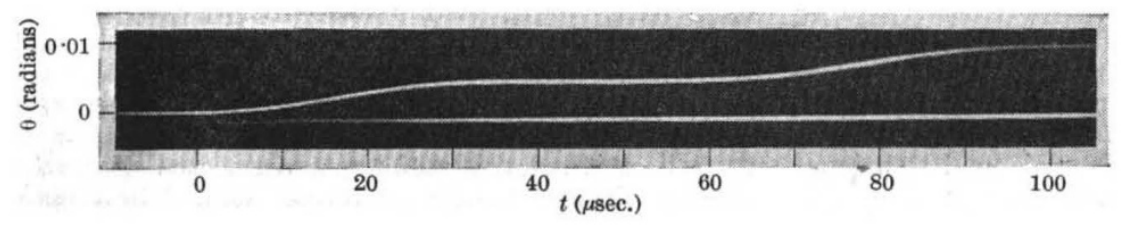

The time-scale of the record was calculated from the geometry of the apparatus and the speed of rota. tion of the turbine, which, in turn, was measured by determining the frequency of the signal from a photo. cell illuminated by a beam of light reflected from the rotating mirror.

The light source used in the experiments was a mercury flash tube energized by the discharge of a condenser. The duration of the flash was arranged to be about 100 microsec., and the flash was synchronized with the arrival of the stress-wave at the record. ing point by a ring-switch and thyratron circuit similar to that described in another connexion ${ }^{2}$.

One of us (J. D. O.) is indebted to the Department of Scientific and Industrial Research for a maintenance allowance.

Department of Physics,

University College of Wales, Aberystwyth. May 31.

'See, for example, Beams, J. W., "Reports on Progress in Physics", 8, 31 (1941)

${ }^{2}$ Davies, R. M., Phil. Trans. Roy. Soc., A, 240, 375 (1948).

\section{Band Systems of Zirconium Oxide ( $\mathrm{ZrO}$ ) in the Ultra-Violet}

THReE new systems of ZrO have been observed in the ultra-violet, in addition to the three triplet systems in the visible region analysed by Miss Lowater. ${ }^{1}$ Lowater also observed twelve bands, de. grading to the longer wave-length, in the region 3726-3470 A., differing in general appearance from the triplet systems in the visible, the bands being much weaker and the triplets not easily recognizable. Although thirty-one bands have been measured in the ultra-violet by us, only five of the twelve band. heads measured by Lowater agree with ours. As the atomic spectrum of zirconium in this region is particularly strong, and as the lines are numerous, weak lines have apparently been mistaken for bandheads. However, it has been possible to photograph the $\mathrm{ZrO}$ spectrum in the region 2930-6700 A. remarkably free from lines, and to identify the real band. heads. Altogether thirty-one bands were measured in the region $4000-2930 \mathrm{~A}$., and assigned to one singlet and two triplet systems.

The source was a 110 -volt D.c. arc running at 4 amp. The lower electrode was of copper, and shaped into a cup of thin walls with a neck of $1.5 \mathrm{~mm}$. at the bottom. This was filled with zir. conium fluoride and made the anode. The cathode was a thick copper electrode flattened at the bottom to effect rapid cooling. The temperature in the anode cup was kept just below the temperature of rapid oxidization. Vapours rising from the molten zirconium fluoride in the anode cup showed strong bands of $\mathrm{ZrO}$ on oxidization just below the cathode. The atomic spectrum of zirconium in this source was very weak. The following systems were photographed.

(1) The $\lambda 3682$ and $\lambda 3589$ bands, now forming the $(0,0)$ and $(2,1)$ bands of the singlet system, were measured by Miss Lowater. The $\lambda 3682$ band has also been observed in the spectra of the late-type 